\title{
The Evaluation of CD3, CD 5, CD10, CD 19, and CD20 Markers in the Differential Diagnosis of the Lymphoma Subtypes in Sudanese Patients
}

\author{
Mohamed Elfatih Abdelwadoud ${ }^{1}$, Nabawia S Ahmed ${ }^{1}$, Hadia Alhaj ${ }^{1}$, \\ Hisham Ali Waggiallah" ${ }^{2 *}$ \\ ${ }^{1}$ Department of Histopathology and Cytopathology, Faculty of Medical Laboratory Sciences, \\ University of Medical Sciences and Technology, Khartoum, Sudan. \\ ${ }^{2}$ Department of Medical Laboratory Science, College of Applied Medical Science, Prince Sattam Bin \\ Abdulaziz University, Alkharj, KSA
}

\begin{abstract}
The objective of this paper was to evaluate the use of CD3, CD5, CD10, CD19, and CD20 markers in the differential identification of lymphoma subtypes.

Methods and Results: This was a retrospective cross-sectional study included 82 patients with palpable lymphadenopathies. The formalin-fixed paraffin block sections immunostained with the Dako flex were investigated. CD3, CD5, CD10, CD19, and CD20 staining was performed on sections. The current study found that the two main types of lymphoma, Hodgkin's lymphoma, amd non-Hodgkin's lymphoma, have a significant association with CD3, CD10, and CD19, and a highly significant association with CD20, implying that these CD markers are crucial for general classification and diagnosis of lymphoma. CD3 had a highly significant relationship with gender. CD3 and CD20 were demonstrated to have a significant relationship with the lymphoma subtypes. The CD20 marker is the most consistent and useful marker for differentiating lymphoma subtypes.(International Journal of Biomedicine. 2021;11(4):493-497.)
\end{abstract}

Key Words: Hodgkin's lymphoma • non-Hodgkin’s lymphoma • immunohistochemistry $\bullet$ CD markers

For citation: Abdelwadoud ME, Ahmed NS, Alhaj H, Waggiallah HA.The Evaluation of CD3, CD 5, CD10, CD 19, and CD20 Markers in the Differential Diagnosis of the Lymphoma Subtypes in Sudanese Patients. International Journal of Biomedicine. 2021;11(4):493-497. doi:10.21103/Article11(4)_OA14

\section{Introduction}

Lymphomas are a group of disorders produced by malignant cells that aggregate in lymph nodes, resulting in the clinical symptoms of lymphadenopathy. They occasionally spread into the blood ('leukemic phase') or invade organs other than lymphoid tissue. The characteristic multinucleated, polyploidy Reed-Sternberg (RS) cells are essential for distinguishing the four classic subtypes, and mononuclear Hodgkin $(\mathrm{H})$ cells are also found in the malignant clone. RS cells and their mononuclear variants ( $\mathrm{H}$ cells) exhibit inconsistency

*Corresponding author: Dr. Hisham Ali Waggiallah, Department of Medical Laboratory Sciences, College of Applied Medical Sciences, Prince Sattam Bin Abdulaziz University, Alkharj, KSA.E-mail: hishamwagg30@hotmail.com in antigen expression. ${ }^{(1)}$ The use of CD markers in medicine has improved our knowledge of a variety of hematological disorders. CD markers are monoclonal antibodies that bind leukocyte cell-surface molecules as well as antigens from other cells. CD5 is found in chronic lymphocytic leukemia/ small lymphocytic lymphoma (CLL/SLL) and mantle cell lymphoma (MCL), while CD10 is present in acute precursor B- and T-cell lymphoblastic leukemia/lymphoma (ALL) and lymphomas of follicular center cell origin. ${ }^{(2)}$

T-cell processes are typically CD $3+, \mathrm{CD} 20-$, and $\mathrm{CD} 45+$, whereas B-cell processes are CD3-, CD20+, and CD45+. Other $\mathrm{CD}$ markers are utilized to distinguish lymphomas. $\mathrm{CD}$ markers allow hematologists to combine immunophenotyping with clinical observations to make an accurate diagnosis. ${ }^{(3)}$

In around $35 \%-40 \%$ of nodular sclerosis and mixed cellularity cases, RS-H cells express the B-cell 
antigens CD19 and CD20.(4,5) Hodgkin's lymphoma (HL) immunohistochemistry reveals that nodular lymphocytepredominant HL (NLPHL) is a distinct HL subtype. This subtype's RS-H cells, defined as lymphocyte and histiocyte $(\mathrm{L} \& \mathrm{H})$ variants, have a distinct polylobated, "popcorn" look and regularly express B-cell markers such as CD20 and CD45 (leukocyte common antigen). ${ }^{(6)}$

There have been multiple occurrences of HL coexisting with non-Hodgkin's lymphoma (NHL), either as sequential occurrences or in the same site, when they have been termed as composite. The most frequent link is lymphocyte predominance and large cell lymphoma. ${ }^{(7)}$ Follicular lymphoma is frequently associated with other subtypes of HL. ${ }^{(8)}$ The objective of this paper was to evaluate the use of CD3, CD5, CD10, CD19, and CD20 markers in the differential identification of lymphoma subtypes.

\section{Materials and Methods}

This was a retrospective cross-sectional study. The research was carried out at Rick Hospital in Khartoum (Sudan), from April to July 2019. Sudanese patients with lymphomas who attended histopathology laboratories agreed to participate in the study after providing written consent.

The sample size was calculated using a convenient sampling technique, and 82 patients with palpable lymphadenopathies were chosen.

Inclusion criteria. All Sudanese patients with lymphadenopathy and diagnosed with any type of lymphomas were enrolled in the study.

Exclusion criteria Participants apparently healthy without signs and symptoms of lymphadenopathy were excluded.

\section{Sample collection and processing}

Tissue sections were prepared on a slide, dewaxed in xylene for two 10-minute shifts, and then hydrated in graded alcohol for two minutes each. After hydration, the slides were stained for 10 minutes with H\&E. Following staining, the slides were rinsed with tap water for 8 minutes before being moved to a coupling jar containing a counterstain, such as Eosin solution, for 2 minutes. The slides were then washed under running water for 1 minute. The stained slides were air-dried and then mounted with DPX. ${ }^{(9)}$ Lymph node paraffin block sections were placed on an adhesive-coated slide containing Poly-L-lysine. After heat-induced epitope retrieval (three minutes at $110^{\circ} \mathrm{C}$ in citrate buffer with $\mathrm{PH}$ 6.0), deparaffinized sections were manually stained with a standard multilink detection kit (Dako detection kit) containing endogenous peroxidase block, nonspecific binding block, horseradish peroxidase, 3,3'-diaminobenzidine as chromogen, and hematoxylin. Five primary polyclonal antibodies were used to stain the sections: The formalin-fixed paraffin block sections were immunostained with the Dako flex, ready-touse antibody system, which has been approved by prominent experts in the field. $.^{(9)} \mathrm{CD} 3, \mathrm{CD} 5, \mathrm{CD} 10, \mathrm{CD} 19$, and CD20 staining was performed on sections (Fig.1). The staining reaction occurred in the nucleus, and the appropriate positive control was applied.

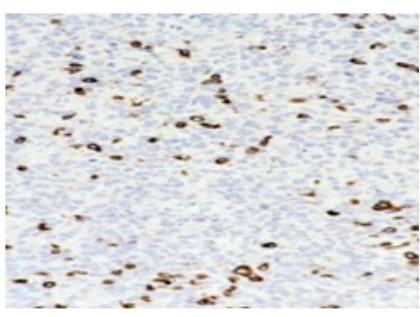

A

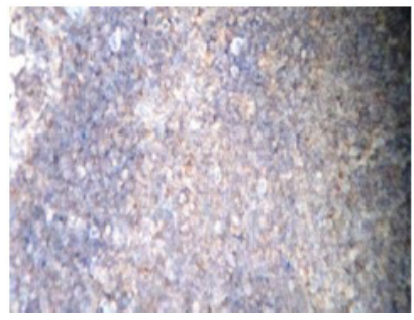

C

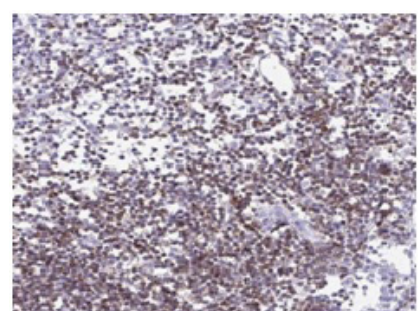

B

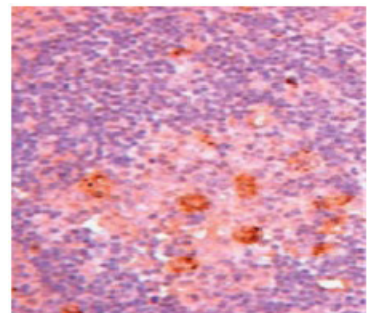

$\mathrm{D}$

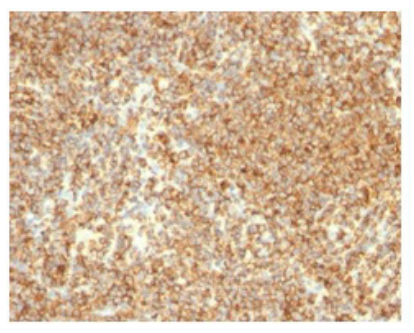

E

Fig. 1. Morphological features of immunohistochmical $C D 3, \quad C D 5, C D 10, C D 19$, and CD20 staining (hematoxylin counterstain) in human lymphoma:

$A-C D 3+; B-C D 5+$; $-C D 10+$ - D- CD19+; E- CD20+

\section{Assessments of results for histopathology and immuno-} cytochemistry

A pathologist at the histopathology department of the hospital where the study was conducted confirmed the malignant cases and their origins once they were diagnosed. H\&E stains and histological immune markers were used to make the diagnosis. The level of staining on positive tumor cells was reported as negative, weak $(1+)$, moderate $(2+)$, or strong $(3+)$. The intensity of strong staining was defined as staining comparable to positive control tissue. Any faint staining in tumor cells was considered weak staining. All tumors that lacked staining of tumor cells were deemed negative. Tumors with +1 staining intensity in up to $10 \%$ of tumor cells were thought to be weakly positive. A +2 intensity in $10 \%-30 \%$ of tumor cells was classified as moderately positive. More than $30 \%$ of tumor cells with a +3 intensity were regarded as a strong positive. To determine sensitivity and specificity, a relatively high cutoff value of $10 \%(1+)$ was used to eliminate false-positive results by using the following formulas:

Sensitivity $=[$ True positive $/$ True positive + False negative $] \times 100$ Specificity $=[$ True negatives $/$ True negative + False positive $] \times 100$

\section{Ethical consideration}

The study was conducted in accordance with ethical principles of the WMA Declaration of Helsinki (1964, ed. 2013) and approved by the Ethics Committee of University 
of Medical Sciences and Technology and Rick Hospital (Khartoum, Sudan). All patients were informed about the purpose and design of the work and gave their consent to participate in the study and publish its results in the open press.

Statistical analysis was performed using the IBM SPSS Statistics for Windows, Version 20.0. (Armonk, NY: IBM Corp.). Baseline characteristics were summarized as frequencies and percentages for categorical variables. Group comparisons were performed using chi-square tests with Yates correction. A probability value of $P<0.05$ was considered statistically significant.

\section{Results}

The total number of participants in this study was 82 lymphoma patients ( $58.5 \%$ men and $41.5 \%$ women). The age ranges were subdivided into four categories. Group 1 included $30(36.6 \%)$ patients under age 20; Group 2 included 14(17.1\%) patients aged between 21 and 40 years; Group 3 included 22(26.8\%) patients between 41-60 years; Group 4 included 16(19.5\%) patients over age 61 (Table 1).

Table 1. Socio-demographic characteristics of the respondents $(n=82)$

\begin{tabular}{|c|c|}
\hline Socio-demographic variables & No (\%) \\
\hline \multicolumn{2}{|c|}{ Age, yrs } \\
\hline $0-20$ & $30(36.6 \%)$ \\
\hline $21-40$ & $14(17.1 \%)$ \\
\hline $41-60$ & $22(26.8 \%)$ \\
\hline$>61$ & $16(19.5 \%)$ \\
\hline \multicolumn{2}{|c|}{ Gender } \\
\hline Male & $48(58.5 \%)$ \\
\hline Female & $34(41.5 \%)$ \\
\hline
\end{tabular}

Table 2 represents the relationship between age groups and gender, and CD markers: CD20 had a highly significant relationship with age groups, and CD3 had a highly significant relationship with gender.

Table 3 represents the relationship between HL/ NHL and CD markers. We found that there is a significant association between HL/NHL and CD3, CD10, and CD19. A highly significant relationship was found between CD20 and lymphoma types.

Table 4 displays lymphoma subtypes and their relationship with $\mathrm{CD}$ markers. $\mathrm{CD} 3$ and $\mathrm{CD} 20$ were demonstrated to have a significant relationship with the subtypes of lymphoma.

\section{Discussion}

As is known, lymphoma is a widespread and difficultto-diagnose disease, so highly sensitive diagnostic techniques had to be used to diagnose it. The current study focused on using $\mathrm{CD}$ markers in a potential diagnostic role in lymphomas as well in their subtypes.

Since each type and subtype displays specific clinicopathologic characteristics, morphological identification and classification of lymphomas depending on immunophenotyping is critical for patient management and prognosis.

Lymphomas are likely to be seen in people of all ages, ranging from 0 to more than 61 years, though they primarily affect those over age 40. According to the current study, CD20 has a significant association with age groups, whereas CD3 has a highly significant association with gender; CD3 expression is rarely abnormal in mature B-cell neoplasms such as diffuse large $\mathrm{B}$-cell lymphoma, classic $\mathrm{HL},{ }^{(10)}$ and follicular lymphoma. CD3 expression in CD20+ B-cell lymphomas is usually not a problem because co-expression of these two markers will trigger further workup with more

Table 2.

Association and frequency of each CD marker in sex and age groups (n=82)

\begin{tabular}{|c|c|c|c|c|c|c|c|c|c|c|}
\hline Groups & \multicolumn{2}{|c|}{ CD3 } & \multicolumn{2}{|c|}{ CD5 } & \multicolumn{2}{|c|}{ CD10 } & \multicolumn{2}{|c|}{ CD19 } & \multicolumn{2}{|c|}{ CD20 } \\
\hline $0-20$ & $22(26.8)$ & $8(9.8)$ & $30(36.6)$ & 0 & $22(26.8)$ & $8(9.8)$ & $28(31.7)$ & $4(4.9)$ & $8(9.8)$ & $22(26.8)$ \\
\hline $21-40$ & $8(9.8)$ & $6(7.3)$ & $14(17.1)$ & 0 & $14(17.1)$ & 0 & $14(17.1)$ & 0 & $8(9.8)$ & $6(7.3)$ \\
\hline$>61$ & $10(12.2)$ & $6(7.3)$ & $16(19.5)$ & 0 & $14(17.1)$ & $2(2.4)$ & $12(14.6)$ & $4(4.9)$ & $4(4.9)$ & $12(14.6)$ \\
\hline$P$-value & \multicolumn{2}{|c|}{0.71} & \multicolumn{2}{|c|}{0.13} & \multicolumn{2}{|c|}{0.12} & \multicolumn{2}{|c|}{0.25} & \multicolumn{2}{|c|}{0.006} \\
\hline \multicolumn{11}{|l|}{ Gender } \\
\hline Male & $40(48.8)$ & $8(9.8)$ & $46(56.1)$ & $2(2.4)$ & $42(51.2)$ & $6(7.3)$ & $40(48.8)$ & $8(9.8)$ & $13(15.9)$ & $35(42.7)$ \\
\hline
\end{tabular}


Table 3.

Association between CD markers and lymphoma types $(n=82)$

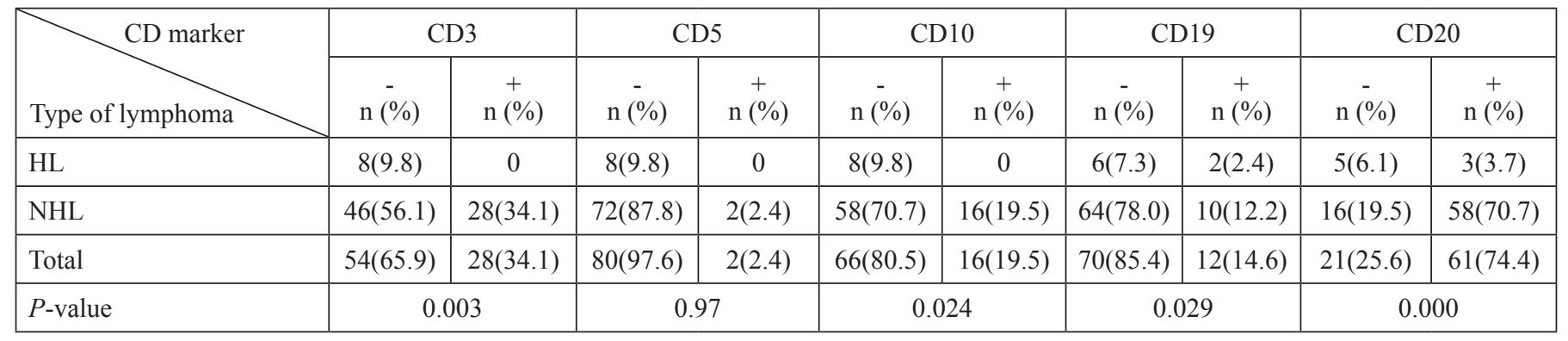

Table 4.

Type of lymphoma and CD markers $(n=82)$

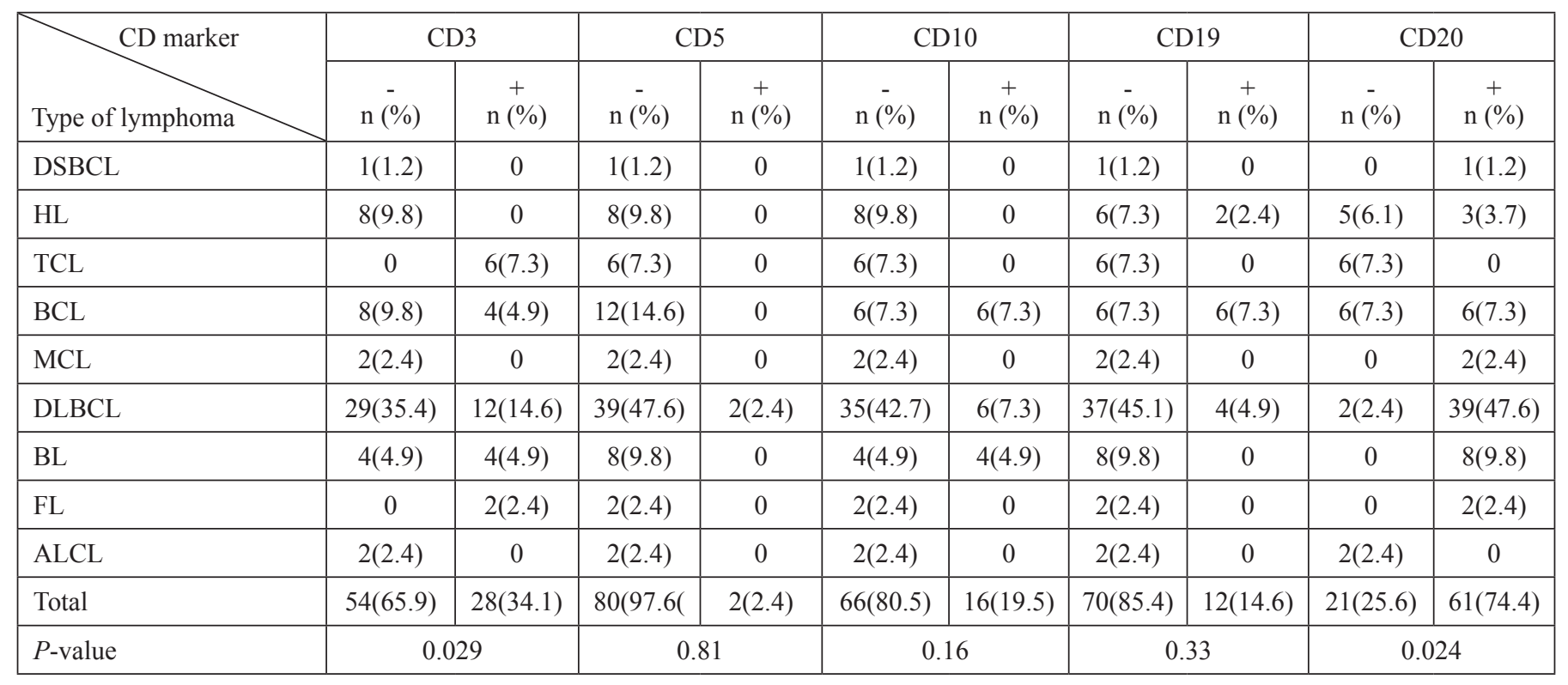

$D S B C L$-diffuse small B cell lymphoma; TCL- T cell lymphoma; BCL - B cell lymphoma; $M C L$ - mantle cell lymphoma; DLBCL-diffuse large $B$ cell lymphoma; BL-Burkitt lymphoma, FL-follicular lymphoma, ALCL - anaplastic large cell lymphoma

lineage-specific markers and molecular studies. ${ }^{(9)}$ Although CD3 expression in B-cell lymphoma can be a diagnostic challenge, lineage identity can be confirmed using a larger panel of immunohistochemical stains, as the CD3 expression is frequently an isolated aberrancy.

About half of all documented instances of CD3-positive DLBCL are EBV-associated large B-cell lymphoma, with plasmablastic or plasmacytic differentiation. In addition to abnormal CD3 expression, EBV-positive DLBCL frequently exhibits down-regulation of B-cell antigens, consistent with plasmablastic or plasmacytic differentiation. ${ }^{(11,12)}$

In the current study, CD5 was found to be a nonsignificant marker in the detection of lymphoma subtypes or even to have an association with age groups or gender, implying that CD5 in Sudanese lymphoma patients is not beneficial as a lymphoma marker. The findings in this study are consistent with previous research that found that CD5 expression abnormalities have been documented in 5\%-10\% of diffuse large B-cell lymphomas (DLBCLs). In the WHO classification (2008) of haematolymphoid neoplasms, CD5+ DLBCL was classified as an aggressive immunophenotypic subtype of DLBCL; however, it was removed from the list of DLBCL subgroups in the revised classification (2016). However, there is considerable debate about the clinical importance of $\mathrm{CD} 5$ expression, and many experts continue to believe that this subpopulation has an exceptionally poor prognosis with frequent therapy failure. ${ }^{(13)}$

The initial stage in immunophenotypic evaluation is determining if the majority cell population is B-cells, T-cells, or neither. Usually, three markers are utilized for this initial classification: CD20, CD3, and CD45. CD20 is the most consistently positive $\mathrm{B}$-cell marker, $\mathrm{CD} 3$ is the most consistently positive T-cell marker, and CD45 is a bone marrow-derived leukocyte marker. T-cell processes are commonly CD3+, CD20and CD45+. Normally, B-cell processes are CD3-, CD20+, and $\mathrm{CD} 45+$. Processes in which the invading leukocytes are either T-cells or B-cells, such as leukemia cutis, are often CD3-, CD20and $\mathrm{CD} 45+.{ }^{(14)}$ The current study found that the two main types of lymphoma, HL and NHL, have a significant association with CD3, CD10, and CD19, and a highly significant association with CD20, implying that these CD markers are crucial for general classification and diagnosis of lymphoma. ${ }^{(14)}$ 
This study demonstrated that the most important CD markers in this research are CD3 and CD20. CD20 has a significant association with lymphoma subtypes, as previously known, and CD20 is expressed on the surface of all B-cells (except early pro-B-cells and plasma cells). The MS4A1 gene on chromosome 11q12.2 encodes the human CD20 molecule. It is involved in B-cell development, maturation, and activation. When bound by a CD20 antibody, the CD20 molecule remains on the membrane of B-cells without dissociation or internalization. CD20 expression varies between lymphoma subtypes. ${ }^{(15,16)}$

In conclusion, the identification of $\mathrm{CD}$ markers not only improves the understanding of pathogenetic mechanisms in lymphomas, but also allows refining classification, improving diagnostic accuracy, and stratifying the outcomes of lymphoma patients, especially when combined with morphological classification. The CD20 marker is the most consistent and useful marker for differentiating lymphoma subtypes.

\section{Acknowledgments}

This publication was supported by the Deanship of Scientific Research at Prince Sattam bin Abdulaziz University.

\section{Competing Interests} interests.

The authors declare that they have no competing

\section{References}

1. Patel HS, Shah S, Goswami HM. Role of Immunohistochemistry in Differential Diagnosis of Lymphoma (A Study of 200 Cases). International Journal of Contemporary Pathology, 2020; 6(1):23-28

2. Dong HY, Gorczyca W, Liu Z, Tsang P, Wu CD, Cohen P, Weisberger J. B-cell lymphomas with coexpression of CD5 and CD10. Am J Clin Pathol. 2003 Feb;119(2):218-30. doi: 10.1309/u98advkuc26r2rja.

3. Campbell SM, Peters SB, Zirwas MJ, Wong HK. Immunophenotypic diagnosis of primary cutaneous lymphomas: a review for the practicing dermatologist. J Clin Aesthet Dermatol. 2010 Oct;3(10):21-5.

4. Hsu SM, Tseng CK, Hsu PL. Expression of p55 (Tac) interleukin-2 receptor (IL-2R), but not p75 IL-2R, in cultured H-RS cells and H-RS cells in tissues. Am J Pathol. 1990 Apr;136(4):735-44.
5. Schmid C, Pan L, Diss T, Isaacson PG. Expression of B-cell antigens by Hodgkin's and Reed-Sternberg cells. Am J Pathol. 1991 Oct;139(4):701-7.

6. Pinkus GS, Said JW. Hodgkin's disease, lymphocyte predominance type, nodular--further evidence for a B cell derivation. L \& H variants of Reed-Sternberg cells express L26, a pan B cell marker. Am J Pathol. 1988 Nov;133(2):211-7.

7. Herbst H, Dallenbach F, Hummel M, Niedobitek G, Pileri S, Müller-Lantzsch N, Stein H. Epstein-Barr virus latent membrane protein expression in Hodgkin and Reed-Sternberg cells. Proc Natl Acad Sci U S A. 1991 Jun 1;88(11):4766-70. doi: 10.1073/pnas.88.11.4766.

8. Gonzalez CL, Medeiros LJ, Jaffe ES. Composite lymphoma. A clinicopathologic analysis of nine patients with Hodgkin's disease and B-cell non-Hodgkin's lymphoma. Am J Clin Pathol. 1991 Jul;96(1):81-9. doi: 10.1093/ajcp/96.1.81. 9. Pan Z, Chen M, Zhang Q, Wang E, Yin L, Xu Y, Huang Q, Yuan Y, Zhang X, Zheng G, Yuan J. CD3-positive plasmablastic B-cell neoplasms: a diagnostic pitfall. Mod Pathol. 2018 May;31(5):718-731. doi: 10.1038/modpathol.2017.177.

10. Wu B, Vallangeon B, Galeotti J, Sebastian S, Rehder C, Wang E. Epstein-Barr virus-negative diffuse large B cell lymphoma with aberrant expression of CD3 and other T cellassociated antigens: report of three cases with a review of the literature. Ann Hematol. 2016 Oct;95(10):1671-83. doi: 10.1007/s00277-016-2749-0.

11. Lee M, Cha HJ, Yoon DH, Suh C, Huh J. EBV-positive diffuse large B-cell lymphoma of the elderly with aberrant expression of CD3 and TIA-1. Blood Res. 2013 Jun;48(2):15660. doi: $10.5045 / \mathrm{br} .2013 .48 .2 .156$.

12. Sun J, Medeiros LJ, Lin P, Lu G, Bueso-Ramos CE, You MJ. Plasmablastic lymphoma involving the penis: a previously unreported location of a case with aberrant CD3 expression. Pathology. 2011 Jan;43(1):54-7. doi: 10.1097/ PAT.0b013e328340bbba.

13. Na HY, Choe JY, Shin SA, Kim HJ, Han JH, Kim HK, $\mathrm{Oh} \mathrm{SH}$, Kim JE. Characteristics of CD5-positive diffuse large B-cell lymphoma among Koreans: High incidence of BCL2 and MYC double-expressors. PLoS One. 2019 Oct 23;14(10):e0224247. doi: 10.1371/journal.pone.0224247.

14. Campbell SM, Peters SB, Zirwas MJ, Wong HK. Immunophenotypic diagnosis of primary cutaneous lymphomas: a review for the practicing dermatologist. J Clin Aesthet Dermatol. 2010 Oct;3(10):21-5.

15. Khandakar B, Wang W, Li S. Primary splenic red pulp diffuse large B-cell lymphoma with anaplastic features. Stem Cell Investig. 2016 Apr 6;3:9. doi: 10.21037/sci.2016.03.04. 16. Katchi T, Liu D. Diagnosis and treatment of CD20 negative B cell lymphomas. Biomark Res. 2017 Feb 7;5:5. doi: 10.1186/s40364-017-0088-5. 